\title{
Comment devenir docteur en sociologie sans posséder le métier de sociologue?
}

\section{Bernard Lahire}

\section{OpenEdition}

\section{Journals}

Édition électronique

URL : http://journals.openedition.org/ress/629

DOI : $10.4000 /$ ress.629

ISSN : $1663-4446$

Éditeur

Librairie Droz

Édition imprimée

Date de publication : 1 avril 2002

Pagination : 41-65

ISBN : 2-600-00679-6

ISSN : 0048-8046

\section{Référence électronique}

Bernard Lahire, "Comment devenir docteur en sociologie sans posséder le métier de sociologue? », Revue européenne des sciences sociales [En ligne], XL-122 | 2002, mis en ligne le 12 décembre 2009, consulté le 19 avril 2019. URL : http://journals.openedition.org/ress/629 ; DOI : 10.4000/ress.629 


\title{
Bernard LAHIRE
}

\section{COMMENT DEVENIR DOCTEUR EN SOCIOLOGIE SANS POSSÉDER LE MÉTIER DE SOCIOLOGUE? ${ }^{1}$}

\begin{abstract}
RÉSUMÉ
Peut-on devenir docteur en sociologie sans avoir acquis les compétences constitutives du métier de sociologue? Une telle interrogation peut paraître provocatrice. Or, l'examen rigoureux et détaillé d'une thèse soutenue le 7 avril 2001 à l'université de Paris V, sous la direction de Michel Maffesoli (G. Elizabeth Hanselmann-Teissier, Situation épistémologique de l'astrologie à travers l'ambivalence fascination/rejet dans les sociétés postmoderne), conduit malheureusement à émettre une réponse positive à une question apparemment saugrenue. L'objectif premier de cet article est d'apporter les multiples preuves de l'absence de sociologie (de point de vue sociologique, de problématique, de rigueur conceptuelle, de dispositif de recherche débouchant sur la production de données empiriques...) dans la thèse en question. Mais le jugement sur ce cas précis fait apparaître, en conclusion, l'urgence qu'il y a à engager une réflexion collective sur les conditions d'entrée dans le métier de sociologue.
\end{abstract}

Le samedi 7 avril 2001, Madame G. Elizabeth Hanselmann-Teissier (connue publiquement sous le nom d'Elizabeth Teissier) soutenait une thèse de sociologie (intitulée Situation épistémologique de l'astrologie à travers l'ambivalence fascination/rejet dans les sociétés postmodernes) à l'Université Paris V, sous la direction de Michel Maffesoli ${ }^{2}$. Les membres présents de son jury - il s'agissait,

1 Je tiens à remercier Stéphane Beaud et Christine Détrez pour la lecture des premières versions de ce texte, ainsi que Charles Soulié pour les données sur les thèses soutenues en sociologie entre 1989 et 1995 qu'il m'a communiqué.

2 Ce n'était pas la première fois que M. Maffesoli faisait soutenir une thèse en rapport avec l'astrologie. Ainsi, en 1989, S. Joubert a soutenu une thèse de doctorat intitulée Polythéisme des valeurs et sociologie: le cas de l'astrologie à l'Université de Paris V, sous sa direction. Le résumé de cette thèse manifeste un style d'écriture d'une aussi douteuse clarté que celui que l'on découvre dans la thèse d'Élizabeth Teissier: «Les figures polythéistes loin d'être les images obsolètes d'un passé primitif ou révolu, sont l'expression d'un agencement métaphorique dans lequel le face-à-face des dieux témoigne d'un éclatement des valeurs dont il s'agit de reconnaître toute la puissance heuristique. Le polythéisme des valeurs pousse la connaissance vers une épistémologie psychomythique qui semble aujourd'hui sensibiliser, tant les sciences exactes que les sciences humaines; en congruence avec cette mutation épistémologique émergent des indicateurs sociaux qui confirment la transformation paradigmatique à l'œuvre dans la post-modernité, telle l'astrologie dont le succès que l'on connaît tend à exacerber certaines valeurs comme la synthèse, le holisme, l'interdisciplinarité, la complexité, l'analogie, la synchronicité, etc... En toile de fond de cet engouement 
outre son directeur de thèse, de Serge Moscovici ${ }^{3}$, Françoise Bonardel ${ }^{4}$ et Patrick Tacussel $^{5}$ (Gilbert Durand ${ }^{6}$ s'étant excusé de ne pouvoir être présent et Patrick Watier ${ }^{7}$ n'ayant pu se rendre à la soutenance en raison de grèves de train) - lui ont accordé la mention «Très honorable». Cette mention est la plus haute qu'un candidat puisse recevoir et le fait qu'elle ne soit pas assortie des félicitations du jury n'ôte rien à l'appréciation très positive qu'elle manifeste (de nombreux universitaires rigoureux ne délivrant la mention «très honorable avec les félicitations » que dans les cas de thèses particulièrement remarquables). Deux professeurs avaient préalablement donné un avis favorable à la soutenance de cette thèse sur la base d'une lecture du document: Patrick Tacussel et Patrick Watier. Formellement, Madame Elizabeth Teissier est donc aujourd'hui docteur en sociologie de l'université de Paris V et peut - entre autres choses - prétendre, à ce titre, enseigner comme chargée de cours dans les universités, solliciter sa qualification afin de se présenter à des postes de maître de conférences ou déposer un dossier de candidature à un poste de chargée de recherche au CNRS.

Une lecture rigoureuse et précise de la thèse dans son entier (qui fait environ 900 pages $^{8}$ si l'on inclut l'annexe intitulée «Quelques preuves irréfutables en faveur de l'influence planétaire », p. XII-XL) conduit à un jugement assez simple: la thèse d'E. Teissier n'est, à aucun moment ni en aucune manière, une thèse de sociologie. Il n'est pas même question d'un degré moindre de qualité (une «mauvaise» thèse de sociologie ou une thèse «moyenne»), mais d'une totale absence de point de vue sociologique, ainsi que d'hypothèses, de méthodes et de «données empiriques » de nature sociologique.

Ce sont les différents éléments qui nous conduisent à ce jugement que nous voudrions expliciter au cours de cet article en faisant apparaître que la thèse 1) ne fait que développer un point de vue d'astrologue et 2) est dépourvue de tout ce qui caractérise un travail scientifique de nature sociologique (problématique, rigueur conceptuelle, dispositif de recherche débouchant sur la production de données empiriques...). Enfin, nous conclurons sur le fait que, s'il vaut la peine de faire l'analyse critique de cette thèse, c'est parce que celle-ci n'a rien d'anodin ou d'anecdotique et qu'elle remet gravement en cause la crédibilité scientifique de la sociologie et de tous les sociologues qui font leur métier et forment les étudiants avec toute la rigueur requise: si c'est bien la personnalité d'une astrologue connue des médias qui a été à l'origine de l'intérêt public porté à la soutenance, un tel évé-

populaire se dessine plus ou moins nettement un certain nombre d'enjeux essentiels un peu comme si l'anodin servait à l'occasion de miroir reflétant à contre-jour les orientations que la post-modernité se donne à elle-même.» (Source: Docthese 1998/1). La thèse vient de paraître (L' homme d'aujourd' hui et les autres : fascination et rejet, Paris, Plon, 2001).

Directeur d'études à l'EHESS (psychologie sociale).

Professeur de philosophie à l'Université de Paris I.

Professeur de sociologie à l'Université de Montpellier III.

Professeur émérite à l'Université de Grenoble II, Fondateur du Centre de Recherche sur l'Imaginaire. Professeur de sociologie à l'Université de Strasbourg II.

8 Un tel travail de lecture demande beaucoup de temps et porte plus que l'ombre du doute sur les lectures d' «un jour», comme celle d'Alain Touraine, qui affirme ainsi: «Je me suis présenté le premier et j'ai consacré la journée du 15 mai à sa lecture» («De quoi Élizabeth Teissier est-elle coupable?», Le Monde daté du 22 mai 2001). 
nement pose au fond la question plus générale du fonctionnement collectif de notre discipline.

\section{UN POINT DE VUE D'ASTROLOGUE}

Que l'astrologie (l'existence bien réelle d'astrologues), les modes d'usage et les usagers (à faible ou forte croyance) de l'astrologie constituent des faits sociaux sociologiquement étudiables, que l'on puisse rationnellement (et notamment sociologiquement ou ethnologiquement, mais aussi du point de vue d'une histoire des savoirs) étudier des faits scientifiquement perçus comme irrationnels, qu'aucun sociologue n'ait à décider du degré de dignité des objets sociologiquement étudiables (en ce sens l'astrologie comme fait social est tout aussi légitimement étudiable que les pratiques sportives, le système scolaire ou l'usage du portable), qu'un étudiant ou une étudiante en sociologie puisse prendre pour objet d'étude une réalité par rapport à laquelle il a été ou demeure impliqué (travailleur social menant une recherche sur le travail social, instituteur faisant une thèse de sociologie de l'éducation, sportif ou ancien sportif pratiquant la sociologie du sport...), ne fait à nos yeux aucun doute et si les critiques adressées à Michel Maffesoli et aux membres du jury étaient de cette nature, nul doute que nous nous rangerions sans difficulté aux côtés de ceux-ci. Tout est étudiable sociologiquement, aucun objet n'est a priori plus digne d'intérêt qu'un autre, aucun moralisme ni aucune hiérarchie ne doit s'imposer en matière de choix des objets ${ }^{9}$, seule la manière de les traiter doit compter.

Mais de quelle manière E. Teissier nous parle-t-elle d'astrologie tout au long de ses 900 pages? Qu'est-ce qui oriente et structure son propos? La réponse est assez simple, car il n'y a aucune ambiguïté possible sur ce point: le texte d'E. Teissier manifeste un point de vue d'astrologue qui défend sa «science des astres » du début jusqu'à la fin de son texte, sans repos. Et pour ne pas donner au lecteur le sentiment d'un parti-pris déformant, nous multiplierons les extraits tirés du texte de la thèse en indiquant entre parenthèses la référence des pages (afin de donner la possibilité de retourner aisément au texte) $)^{10}$.

\section{Des commentaires astrologiques}

La première caractéristique notable de cette thèse est l'absence de distance vis-à-vis de l'astrologie. On y découvre de nombreux commentaires astrologiques sur des personnes, des événements, des époques. Par exemple, sous le titre «Application de la méthode astrologique: l'analyse du ciel natal d'André Malraux », les pages 120 à 131 de la thèse relèvent clairement d'une «analyse

9 C'est pour cela que nous ne pouvons pas suivre Jean Copans ( « La sociologie, astrologie des sciences sociales?», Le Monde daté du 30. 04. 01) lorsqu'il fait porter la critique sur les objets jugés trop futiles («Les incivilités dans le 93 », «Mon portable, mon ordinateur et ma belle-fille», «L'interculturel entre la rue des Rosiers et le quartier de la Rose»).

10 Tout ce que nous mettons entre guillemets dans ce texte sont des extraits de la thèse. Les italiques sont des choix de soulignement de l'auteur de la thèse et les gras sont nos propres soulignements de lecteur. 
astrologique» de la destinée de l'écrivain et ancien ministre («plutonien grand teint»). M. Weber est qualifié de «taureau pragmatique» (p. 38) et l'on «apprend» diversement que G. Simmel est «Poisson», que W. Dilthey est «Scorpion», que le psychologue C. G. Jung est «Lion» (p. 250), que l'ancien PDG d'Antenne 2, Marcel Jullian, est «Verseau», etc. À chaque fois l'auteur, nous gratifie d'une analyse mettant en correspondance le «ciel natal» de la personnalité et sa pensée:

«Par ailleurs, nous découvrîmes que, par exemple, les systèmes philosophiques et religieux étaient en correspondance avec leurs auteurs via leurs personnalités. [...] Autrement dit, qu'ils étaient hautement relatifs et ne pouvaient être qu'à l'image de leurs concepteurs, résultante d'un regard unique, celui de leur ciel natal.» (p. XI)

«L'astrologue n'est pas étonné de constater une amusante convergence entre ce côté 'flottant', mouvant, quelque peu imprécis ou fantasque et les Poissons, signe astrologique de Simmel; le signe par excellence, avec le cancer (autre signe d'eau) [...] de la mobilité adaptable, de la rêverie, du sens de l'illimité et du cosmique, d'une intuition fine et sensorielle. Signe double de surcroît, reflétant la dualité fondamentale de la philosophie simmelienne [...]. C'est à ses planètes en Verseau que Simmel doit son goût pour l'altérité, la communication, mais aussi son originalité, son amour du paradoxe et sa nature imprévisible.» (note 47, p. 34)

«Petit clin d'œil de l'astrologue: Dilthey, créateur d'une nouvelle théorie de la connaissance fondée sur la compréhension, né le 19 novembre 1833, était Scorpion et théologien de formation...» (note 91, p. 61)

«après avoir démontré par un exemple concret (l'analyse du ciel natal d'André Malraux) l'application pratique, venons-en à son histoire » (p. 132)

«Puisqu'il s'agit ici de rendre également compte d'une expérience personnelle... en effet, c'est à l'âge de 14 ans et demi (à la mi-temps du cycle de Saturne, planète de la réalisation de soi, surtout pour le Saturnien qu'est le Capricorne) qu'est né notre éveil pour l'astrologie.» (p. 288)

«Elle [1'astrologie] participe de cette mutation culturelle, scientifique, philosophique et morale de notre époque [...] au même titre que l'idée de solidarité et de fraternité libertaire incluses dans le symbolisme du Verseau.» (p. 509)

E. Teissier est d'ailleurs très claire quant à la primauté de l'explication astrologique sur tout autre point de vue (dont le point de vue sociologique qu'elle est censée mettre en ouvre dans le cadre d'une thèse de sociologie) pour comprendre les faits sociaux. Critiquant une citation de Serge Moscovici qui évoque les causes sociales des crises, elle écrit: «il nous semble qu'il occulte en l'occurrence la dimension cosmique desdits phénomènes; une dimension qui, selon le paradigme astrologique - et notre conviction - vient coiffer le social. En effet, le social est loin d'expliquer toutes les 'crises... qui se produisent dans la société'. À preuve les actions totalement illogiques, non linéaires, non-logiques et inexplicables autrement que par le paramètre astral qui joue alors le rôle de paramètre éclairant et englobant coiffant le non-logique apparent.» (p. 525). C'est l'astrologie qui explique les faits psychologiques, sociaux et historiques:

[Dans le cadre d'une partie intitulée «La cyclicité planétaire», p. 265-271] «Mais il va de soi que ce sont les mêmes astres avec leurs harmonies et leurs dissonances qui jouent sur les destins individuels.» (p. 268); «Jusqu'au jour où nous réalisons que le 2 décembre correspond à une position de Soleil de $9-10^{\circ}$ en Sagittaire, qui se trouvait très impliquée 
dans le thème de Napoléon Bonaparte» (p. 269); «Le mystère s'éclaircit dès lors que l'on a recours au sésame astrologique: hasard exclu !»; «Quant aux songes répétitifs, ils s'expliquent par l'angle que fait Neptune (rêves) avec ce même point en Scorpion (10 novembre). CQFD» (p. 270)

«Cette lettre et notre réponse, reproduites in extenso [...] sont aussi un exemple significatif du désarroi psychologique dans lequel peuvent nous plonger certaines dissonances planétaires » (p. 321)

«C'est ainsi que nous avons été en mesure de prévoir, entre autres, le krach boursier du 19 octobre 1987, ainsi que de nombreuses turbulences boursières exceptionnelles, souvent assimilées à des mini-krachs...» (p. 432)

«Signalons que, pour l'astrologue, cette période de convulsions sociologiques et philosophiques ne s'inscrit pas dans le hasard, mais se trouve reflétée par les grands cycles cosmiques.» (p. 830)

Et c'est E. Teissier qui conclut elle-même son premier tome par un lapsus (sociologiquement compréhensible) ou un aveu, comme on voudra, consistant à parler de sa réflexion comme relevant d'un travail d'astrologue et non de sociologue: «Le travail de l'astrologue sera maintenant d'interpréter ces données, de tenter aussi de les expliquer. Et ce, ainsi que nous sommes convenus depuis notre étude, à travers l'outil de la compréhension. Rappelons-nous en quels termes Weber définit la sociologie dans Wirtschaft und Gesellschaft...» (p. 463)

L'astrologie est à ce point structurante du propos que, bien souvent, la manière dont E. Teissier conçoit son rapport à la sociologie consiste à puiser dans les textes de sociologues des éléments qui lui «font penser» à ce que dit ou fait l'astrologie. Dans la sociologie, une astrologie sommeille:

[À propos de la notion astrologique d'interdépendance universelle] «Une notion qui, en sociologie, peut être rapprochée du Zusammenhang des Lebens (liaison du vécu au quotidien) de Dilthey, d'une cohérence de la vie où chaque élément est pris en compte et complète le donné social» (p. XIV)

«À noter que la typologie zodiacale rappelle la théorie wébérienne de l'idéal-type, dans la mesure où chaque signe correspond au prototype purement théorique d'une personnalité, en liaison avec le symbolisme du signe.» (p. 248)

\begin{abstract}
«Plus concrètement, cette empathie, pierre angulaire de la consultation, oblige l'astrologue à se mettre à la place de son consultant, d'entrer en quelque sorte dans sa peau afin de comprendre son fonctionnement psychologique. On peut en l'occurrence transposer ici la parole de Weber, afférente à la démarche cognitive de l'observateur en sociologie. [...] En réalité, cette sympathie, cette empathie par rapport à l'expérience d'autrui, une des clefs de la sociologie compréhensive, est aussi le sésame de tout praticien, dont l'objet est la psyché humaine. L'idéal-type de l'astrologue devra, même si c'est difficile, satisfaire à ces conditions opérationnelles de la consultation en engageant la totalité de son être.» (p. 390)
\end{abstract}

\title{
Point de vue normatif et envolées prophétiques
}

Le point de vue sociologique n'est pas un point de vue normatif porté sur le monde. Le sociologue n'a pas, dans son étude des faits sociaux, à dire le bien et le mal, à prendre partie ou à rejeter, à aimer ou à ne pas aimer, à faire l'éloge ${ }^{11}$ ou à

11 M. Maffesoli, «Éloge de la connaissance ordinaire», Le Monde daté du 24 avril 2001. 
condamner. En l'occurrence, une sociologie de tel ou tel aspect du «fait astrologique » ne doit en aucun cas se prononcer en faveur ou en défaveur de l'astrologie, dire si c'est une bonne ou une mauvaise chose. Or, Elizabeth Teissier demeure en permanence dans l'évaluation normative des situations, des personnes et des points de vue, prouvant qu'elle écrit en tant qu'astrologue et non en tant que sociologue des pratiques astrologiques. Ce jugement normatif se manifeste, comme nous le verrons tout au long de ce rapport de lecture, à différents niveaux:

1) Dans l'évaluation positive (défense) de l'astrologie. De ce point de vue, tous les moyens sont bons pour prouver l'intérêt de l'astrologie. E. Teissier se sert de façon générale de la légitimité des «grands » qui auraient accordé de l'intérêt pour l'astrologie ${ }^{12}$, quelle que soit la nature de leur «grandeur» (elle peut ainsi tout aussi bien citer Balzac, Goethe, Fellini, Thomas d'Aquin, Bacon, Newton, Kepler, Einstein, Jung, Laborit, le roi Juan Carlos d'Espagne ou l'ancien Président François Mitterrand): politique, cinématographique, philosophique, littéraire et, bien sûr, scientifique.

2) Dans l'évaluation négative de la partie des astrologues jugés peu sérieux, mais aussi de la voyance et autres pratiques magiques. Si E. Teissier ne se prive pas d'être dans le jugement positif à l'égard de l'astrologie qu'elle qualifie de «sérieuse», elle n'hésite pas à porter un regard négatif sur les autres pratiques. En portant de telles appréciations, elle se comporte alors en astrologue en lutte pour le monopole de la définition de l'astrologie légitime, et nullement en sociologue: «Qu'il s'agisse de la presse écrite, de la télévision, du minitel ou d'internet, les horo-
scopes foisonnent, noyés dans un contexte qui, la plupart du temps, n'est qu'une gros-
sière caricature d'ésotérisme - voyance, cartomancie, tarots, numérologie et autres
retours d'affection appartenant à l'univers magico-mystificateur de pratiques paranor-
males» (p. 4)

[Fustigeant ses confrères sur minitel] «rares sont les programmes vraiment sérieux - on peut les estimer à un nombre situé entre 50 et 100 - qui pratiquent une astrologie digne de ce nom. Les autres? Des avatars plus ou moins ludiques, des cocktails habiles et mystificateurs qui usurpent le nom astrologie, leurs concepteurs nourrissant l'espoir que cela leur donnera une coloration peu ou prou scientifique» (p. 74)

«séparer le bon grain (les astrologues compétents) de l'ivraie (les exploiteurs opportunistes d'une crédulité générale latente)» (p. 294).

Critique de la «fast-astrology» d'une «pauvreté parfois consternante» (p. 305).

[Les rédacteurs en chef de magazines ou journaux acceptent de publier] «des prévisions bateau minimalistes et d'un niveau intellectuel souvent consternant» (p. 556)

3) Dans l'évaluation négative des scientifiques (astronomes notamment, mais pas seulement) qui ne veulent pas reconnaître la légitimité de la «science des astres» (cf. infra «L'astrologie victime d'un consensus socioculturel et de la domination de la 'science officielle'»).

12 Nous ne vérifierons pas ici la véracité des sentiments positifs à l'égard de l'astrologie que l'auteur prête à diverses personnalités. 
4) Dans l'évaluation négative de nombres de journalistes ou de médias qui se moquent des astrologues et de l'astrologie (cf. infra «Les 'données': anecdotes de la vie personnelle, médiatique et mondaine d'E. Teissier»).

5) Dans l'évaluation négative d'une partie du public usager des prédictions astrologiques. E. Teissier manifeste un souverain mépris, parfois teinté d'ironie ou d'une extrême condescendance, vis-à-vis d'une partie de son propre publicc ${ }^{13}$ :

[Dans les «appels à l'aide» du courrier, des lecteurs lancent de] «véritables cris d'alarme ou de désarroi jetés par des déprimés las de vivre et de se battre contre l'adversité » et certains «se lancent alors dans un historique englobant toute leur misérable existence et nous gratifient d'une épître interminable couvrant parfois une vingtaine de pages d'une petite écriture serrée et frileuse.» (p. 312)

[À propos d'une lectrice qui lui demande si elle croit aux envoûtements car elle pense que ses voisins marocains l'ont envoûtée et qui lui demande si elle connaît des «sorciers sérieux » qui pourraient l'aider] «Manifestement cette lectrice était affligée d'une confusion intellectuelle - sinon mentale - évidente. Car [...] elle assimilait la pratique astrologique à la magie et à la sorcellerie, auxquelles l'astrologie est totalement étrangère [...]» (p. 372)

[À propos d'un allemand qui lui envoie du courrier depuis 1981, «deux à trois lettres par semaine, parfois des petits paquets contenant des bibelots kitsch»] «Il a ainsi dépensé une véritable fortune en timbres depuis presque vingt ans qu'il s'adonne à cette déviance unilatérale, à ce monologue pervers.» (p. 377)

«Les couches les moins cultivées de la société, ouvriers et agriculteurs, sont peut-être les plus vulnérables à cette fascination globale et non discriminatoire. [...] pour peu qu'une idée soit séduisante et si possible étrange, les gens gobent sans discrimination ce qu'on leur sert, tant est profond le goût du merveilleux et mystérieux le besoin de renouer le dialogue avec l'ordre primordial, avec le cosmos.» (p. 470-471)

À propos de T. W. Adorno qui, dans un ouvrage critique sur l'astrologie, dit que celle-ci participe de l'acceptation par les dominés de l'ordre établi, E. Teissier écrit: «En l'occurrence, à quoi servirait de faire sentir à ces petites gens leur dépendance, de les appeler à la révolte? Peut-être à les rendre plus malheureux encore qu'ils ne sont?» (p. 582); et elle rajoute une page plus loin, sans s'apercevoir qu'elle semble parler d'elle-même: «Bref, le mépris et l'arrogance percent sans arrêt à travers ces textes, de même qu'un esprit dénué totalement de sérénité et d'objectivité.» (p. 583)

Mais de même qu'il ne doit être ni dans l'éloge ni dans la détestation, le sociologue n'étudie que ce qui est et non ce qui sera. Or, E. Teissier annonce l'avenir à de nombreuses reprises, prophétisant ce qu'elle désire ou, comme on dit plus ordinairement, prenant ses désirs pour des réalités (à venir). Si l'astrologue critique la lecture de l'avenir dans le marc de café, elle n'hésite cependant pas elle-même à prédire l'avenir sur la base de ses simples intuitions personnelles:

13 Elle peut soutenir à d'autres moments que la vérité sort de la bouche du peuple, parce que - mythe $\mathrm{du}$ «bon peuple» oblige - celui-ci serait moins perverti par les institutions académiques, culturelles et médiatiques officielles: «Les gens simples, moins victimes d'a priori (il s'agit d'un terrain intellectuel en quelque sorte vierge) sont plus réceptifs, donc plus vrais par rapport à ce genre de constat, de reconnaissance.» (p. 483). Ou encore: «Il est certain que l'instinct populaire, très sûr parce que nourri de toute l'expérience humaine, cet 'inconscient collectif' cher au psychologue C. G. Jung, a depuis toujours l'intuition d'une action du ciel sur ce qui vit sur terre.» (p. XV). 


\begin{abstract}
«Mais notre regard se portera plus loin et tentera de se projeter sur une vision prospective, essayant de pressentir et de supputer, étant donné le contexte sociétal d'aujourd'hui, la probable évolution du phénomène bifide qui nous occupe: y aura-t-il fusion, intégration harmonieuse de cette conjonctionis oppositurum, dans une sorte de synthèse féconde, et alors quelle forme pourrait prendre cette dernière? [...] En d'autres termes, dans quel sens ira, selon toute vraisemblance, cette mouvance sociale ?» (p. 9)

«Nous oserons même tenter une incursion imaginaire dans l'avenir, à la recherche, en quelque sorte, du temps futur et de l'évolution probable du phénomène socio-astrologique» (p. 69)

«Car la raison sèche, la raison ratiocinante a fait son temps. Voici venir l'âge d'une raison ouverte, d'une 'raison plurielle', réconciliée avec la passion et le vital en l'homme, sa libido - ou pulsion vitale - véhiculant à la fois sa sensibilité et son feu intérieur.» (p. 834)
\end{abstract}

«Mais les nouvelles énergies sont en marche, comme l'annonce Abellio, 'l'incendie de la nouvelle science fera irruption dans le monde'» (p. 850)

\title{
L'astrologie est une science, voire la plus grande des sciences
}

Contrairement à certains lecteurs pressés, et empressés de communiquer à la presse le résultat de leur précipitation, qui soutiennent que E. Teissier n'a jamais défendu l'idée que l'astrologie était une science ${ }^{14}$, une lecture exhaustive de la thèse fait apparaître très exactement le contraire. L'auteur parle diversement de la «science des astres » (à de très nombreuses reprises tout au long de la thèse) ou de «la science empirique des astres» (p. 258), de «la science par excellence de la caractérologie» (p. XI), de «la science par excellence de la personnalité» (p. 92 ou 815), de la «science de la qualité du temps» (p. 112), d'une «science empirique par définition» (p. 769) ou de «la reine des sciences» (p. 72) ${ }^{15}$. Parfois l'astrologie est considérée comme une science sociale parmi d'autres, parfois comme une «science de l'esprit» opposée aux «sciences de la nature» ou une «science humaine» (p. 98) opposée à l'astronomie comme «science de l'observation».

«à défaut de pouvoir être classé dans les sciences exactes, s'agit-il d'un savoir à connotation scientifique - fût-ce par le biais des sciences humaines? [...] Pour une large part, celle-ci, en tant que science empirique, est de l'ordre du vérifiable et échappe ipso facto à la notion de 'croyance'. Car l'astrologie, en tant que système culturel cohérent, a pour ambition de déchiffrer le réel à l'aide d'un référentiel universel et permanent - l'alphabet céleste du système solaire - référentiel invariable et donc prévisible dans sa rigueur mathématico-astronomique» (p. 24-25)

«En effet, l'astrologie étant, au même titre que la psychologie, la sociologie ou la religion, une science de l' esprit [...] par opposition à une science de la nature (bien qu'elle englobe celle-ci dans son objet), il n'est pas question ici de faire appel à un positivisme rationaliste expérimental qui ne relèverait que du quantitatif» (p. 27)

«la problématique épistémologique soulevée par la nature des sciences sociales en général et par l'astrologie en particulier» (p. 48)

14 «[... je n'ai lu nulle part dans sa thèse que l'astrologie était scientifique», A. Touraine, « De quoi Élizabeth Teissier est-elle coupable?», op. cit.

15 Elle écrit par ailleurs: «D'autre part, la télépathie ne s'est elle pas imposée comme discipline scientifique depuis les expériences de Rhine?» (p. 281). 
«Selon nous, on l'aura compris, l'astrologie est un système cohérent, mathématiquement rationnel (supra-rationnel, selon Fischler) et vérifiable d'un astrologue à l'autre, ayant pour soubassement les données astronomiques fournies par les observatoires, à l'encontre de pratiques occultes et plus ou moins gratuites.» (p. 579)

Mais on trouve aussi, toujours dans l'ordre de la référence scientifique, des revendications de plus grande dignité et de supériorité. Non seulement l'astrologie est une science, mais c'est la plus haute des sciences:

«On peut dire en somme que sans être classable dans l'une ou l'autre de ces catégories de la connaissance, l'astrologie est une émanation partielle de chacune de ces disciplines qu'elle englobe en un système ambitieux.» (p. 22)

«Elle apparaît de ce fait comme peut-être la seule science objective de la subjectivité, avec ce qu'elle peut contenir d'hénaurme, au sens ubuesque du mot, et de dérangeant.» (p. 250)

«L'astrologie est la mathématique du tout (dans la Rome antique, les astrologues étaient d'ailleurs appelés les mathematici). Elle est holistiquement logique, au contraire d'une logique fragmentaire, linéairement rationnelle.» (p. 501)

«Que connaissaient-ils tous de cette science? Car à nos yeux, c'en était une, une science humaine bien plus charpentée que beaucoup d'autres, qui étaient respectées, elles. D'où venait que la plus vérifiable était justement la plus tabou, la plus salie, la plus rejetée? À croire que la vérité était maudite quelque part.» (p. 597-598)

Il ne faut cependant pas attendre de l'auteur trop de cohérence au sujet de la scientificité de l'astrologie, car elle peut tout aussi bien soutenir à d'autres moments que ce savoir se situe entre le mythe et la science ou qu'il est finalement en lien avec la plupart des sciences humaines et sociales, la philosophie, la poésie, la religion et la mythologie. Cette variété des définitions hétérogènes participe de la volonté de mettre en évidence l'extraordinaire richesse et l'irréductible complexité de l'astrologie:

«S'appuyant sur un langage symbolique en congruence avec tous les niveaux de réalité de L'Etant, et ce aussi bien sur un plan collectif qu'individuel, elle participe avant tout des sciences qui étudient l'homme, comme la philosophie (en particulier la métaphysique, à travers la cosmogonie qu'elle implique), la psychologie, la médecine, la biologie; elle flirte avec la poésie; mais elle est partie prenante également des sciences qui étudient la société humaine et ses produits, comme l'histoire et les sciences politiques (à travers la théorie des cycles), les sciences sociales (à travers les modes, les mouvements collectifs et les mentalités), la prospective (via la prévision qu'elle permet). Et n'oublions pas la religion (en liaison avec son caractère originellement sacré, l'éthique et l'esthétique qu'elle sous-tend), ni, bien entendu la mythologie.» (p. 21).

«Nous verrons que l'astrologie se situe effectivement quelque part entre ces deux univers du mythe immémorial et de la pure scientificité...» (p. 210)

«L'astrologie qui se situe au carrefour de la philosophie métaphysique, de la religion et de la science, qui participe à la fois de l'image et du concept, se place également quelque part entre le sacré et le profane.» (p. 478)

Ailleurs encore, l'astrologie est présentée comme étant presque à l'avantgarde du «Nouvel esprit scientifique» et participant d'une «épistémologie de la complexité ». Non seulement elle est une science, et l'une des plus grandes d'entre elles, mais en plus elle s'avère plus avancée que toutes les autres: 
Le «système astrologique» est «orienté sur la loi hermétique des correspondances, sur l'idée de sympathie universelle, autrement dit sur la notion, essentielle pour le Nouvel Esprit scientifique, d'interdépendance universelle» (p. XIV)

«l'astrologie [...] non seulement ne serait pas en contradiction avec le paradigme du Nouvel esprit scientifique, mais serait au contraire depuis toujours en congruence totale avec ce dernier» (p. 752)

«On peut donc imaginer que la science n'admettra la validité de l'astrologie que lorsqu'elle aura elle-même changé de paradigme en se rangeant du côté du nouvel esprit scientifique et en acceptant de reconnaître la réalité de l'esprit. Car en dernier ressort, la science finira par atteindre ses propres limites en touchant les limites de la matière...» (p. 765)

«cette 'crise de la science' aboutit à une nouvelle Weltansschauung qui ne demande qu'à renaître, celle de la complexité (Morin). Tournant paradigmatique, donc, équivalent à un glissement d'une épistémologie vers une ontologie, cette épistémologie étant celle de la complexité (Morin)» (p. 843).

Mais si l'astrologue est si en avance, c'est - nous explique l'auteur sans rire qu'à la différence de l'astronome «qui a en général une approche purement physique et mécaniste de sa science » et qui «est hypnotisé par la petitesse des astres, leur éloignement, leur faible masse par rapport au soleil», lui, «en écoute la musique» (p. 98). La tristesse du savoir de celui qui «évalue le poids et la matière du disque, ses dimensions et sa température, suppute sa densité » (p. 98) est grande face à la joie de celui qui sait écouter «la musique des sphères, chère déjà à Plotin, avant qu'elle ne fasse rêver Kepler» (p. 98).

\section{L'astrologie victime d'un consensus socioculturel et de la domination de la «science officielle»}

Pourquoi, se demande E. Teissier, l'astrologie ne bénéficie-t-elle pas de la légitimité académique (universitaire) et scientifique (au CNRS)? Sa réponse - formulée à maintes reprises dans le texte - est la suivante: l'astrologie («la science des astres ») est victime d'un rapport de domination qui est parvenu à instaurer un véritable consensus socioculturel en sa défaveur. La science, souvent rebaptisée «science officielle», «pensée unique» ou «conformiste», opprime l'astrologie et fait croire au plus grand nombre qu'il s'agit d'une «fausse science» en cachant la réalité des choses («conjuration du silence», p. 816). La «science officielle» est donc considérée comme une idéologie dominante, un «lieu totalitaire», un «impérialisme» ou un «terrorisme» face à cette «contre-culture» astrologique qui est maintenue dans un véritable «ghetto». Pire encore, la science n'est qu'affaire de «mode» et de «convention» et ne parvient à maintenir sa domination que par un enseignement officiel qui dicte à tous ce qu'il est bon de penser:

\footnotetext{
«Plus ou moins consciemment, nous étions convaincue, à l'instar de toute personne fermée à l'astrologie a priori et par convention, que l'absence de tout enseignement officiel reléguait la science des astres dans les fausses sciences.» (p. IX)

«Presque aussi ahurissante était l'occultation de ce paramètre philosophique dans notre culture occidentale, le fait qu'à travers toutes nos études - jusqu'à vingt-quatre ans jamais nous n'avions entendu parler d'astrologie. Mieux: on nous avait soigneusement caché - comme on continue de le faire - que les plus grands esprits - R. Bacon, St Thomas d'Aquin, Newton, Kepler, Balzac, Goethe, Einstein, Jung... avaient soit pratiqué,
} 
soit vénéré la science des astres. Pourquoi ce parti pris de mise au ban de la plus pérenne des connaissances humaines? Nous prîmes alors conscience de la relativité du consensus intellectuel d'une époque, vouée aux modes, muselée par ses courants de pensée; nous nous apercevions que l'enseignement officiel était un colosse aux pieds d'argile» (p. X)

«un pays, une culture sont le reflet de leur enseignement académique qui dicte ce qu'il convient de penser, le bien penser. La doxa (l'opinion), véhiculée en particulier par les médias, tout en ayant la coloration du sens commun, reste néanmoins sous l'influence de la pensée conformiste qui lui sert de référence» (p. XII-XIII)

«cette allergie aux astres qui débouche sur l'ostracisme culturel face à une contre-culture provocatrice» $(\mathrm{p} . \mathrm{XIII})$

«Les résultats de telles recherches pourraient changer l'actuel consensus socioculturel, entraînant un changement dans les mentalités, et ce notamment au sein d'une certaine intelligentsia que J.-M. Domenach appelle les gens du demi-savoir.» (p. XVI)

«croyance illicite, donc persécutée, ses partisans faisant éventuellement office de bouc émissaire.» (p. 24)

«un certain terrorisme desséché de la pensée scientifique officielle» (p. 25)

«du fait de sa ghettoïsation, le milieu astrologique peut s'inscrire parmi les minorités culturelles » (p. 32)

«lobby scientifique face à l'astrologie» (p. 52)

«rationalisme dominant, lequel se trouve également à la source de la suppression de l'enseignement officiel de l'astrologie » (p. 88)

«C'est seulement au XVIIème siècle que ces deux sciences bifurquent. La mode est désormais à l'astronomie, sœur matérialiste de l'astrologie.» (p. 94-95)

«Et, forte du consensus socio-culturel qui la soutient tel un socle confortable, elle [la science] se permet d'opérer des évaluations [...] et des appréciations [...]» (p. 736)

«La science apparaît comme un lieu totalitaire qu'il ne faut pas remettre en question, où la compétition et le mandarinat jouent un rôle essentiel pour nombre de scientifiques.» (p. 737)

Les «préjugés» et les «clichés» sont ainsi du côté de la «science officielle». Les rationalistes sont «agressifs », «dogmatiques», «attardés » et sont accusés de manque de curiosité pour ne pas vouloir s'intéresser à l'astrologie et, surtout, pour ne pas lui trouver de l'intérêt: «Aujourd'hui, l'obscurantisme, l'opposition aux Lumières n'est plus du côté que l'on croit.» (p. 816).

«la raison en tant que telle n'a-t-elle pas outrepassé ses prérogatives et trahi sa vocation de sereine souveraineté pour se scléroser, tel un vieillard tyrannique?» (p. 7)

« dogme implicite et respecté de la pensée dominante d'une société, en l'occurrence de la nôtre» (p. 11)

«rationalistes agressifs et allergiques aux astres» (p. 42)

«C'est un truisme d'affirmer que les décideurs, les hommes d'affaires sont de grands pragmatistes: ils retiennent ce qui marche, et s'encombrent peu, si les faits le commandent, des préjugés inhibants des rationalistes purs et durs.» (p. 430)

«C'est bien là la problématique de l'astrologie face à la condamnation des rationalistes purs et durs: 1'inadéquation du système rationaliste. Sous cet aspect, on peut sans doute 
se ranger du côté d'Abellio qui traitait ces derniers d'attardés. Lorsque les représentants de cette tendance se retrouvent dans les médias, ils se muent en robots de la pensée, en mercenaires du système rationaliste.» (p. 638)

«Comme on l'a vu dans Duel sur la cinq, le scientifique se retranche derrière ses phrases clés, des phrases à consonance magique, aussi paradoxal que cela puisse paraître pour un savant: 'L'astrologie n'est pas scientifique'... Lorsque cet argument est ainsi récurrent, il fait figure à la fois de défense, d'attaque, de bouclier, à l'instar de l'encre projetée par la seiche. Visiblement on ne veut - on ne peut - accepter l'échange, la discussion, l'argumentation. On fait appel à ce qui ressemble à un véritable credo, celui de la science officielle. Derrière cette attitude on trouve bien évidemment de l'arrogance, du mépris, une condescendance de bon aloi, mais aussi beaucoup de peur; la peur d'être déstabilisé, la peur d'être confronté à un inconnu qu'on ne pourra ni intégrer ni gérer. D'où l'attitude iconoclaste du scientifique positiviste, qui se réfugie dans la déliance (à l'opposé de la reliance), dans un splendide isolement.» (p. 729)

«refus d'expérimenter que l'on constate presque universellement dans le domaine de la science officielle» (p. 756)

\section{L'argument relativiste}

On voit bien qu'invoquant le consensus socio-culturel et la domination, E. Teissier avance les éléments clefs de la position la plus naïvement relativiste. Remplacez les enseignants de physique par des enseignants d'astrologie, appelez l'astrologie la «science des astres » et imposez la à tous ceux qui passent par l'institution scolaire et vous verrez que la théorie de la relativité ne vaut guère mieux que l'analyse astrologique du ciel natal. Tout est affaire de mode et d'imposition purement arbitraire. Tout est relatif.

\footnotetext{
«Nous ne pouvions accepter - ni même envisager - l'idée qu'une société entière, surtout en notre époque postmoderne - donc, pensions-nous, évoluée - pouvait avoir tort, qu'elle était, elle aussi, comme toutes celles qui ont précédé, essentiellement relative.» (p. IX)

«La valeur d'une discipline n'est-elle pas relative à ceux qui la jugent; or, ceux-ci peuvent-ils juger ex nihilo, dégagés de tout a priori, de toute influence, de toute détermination socioculturelle?» (p. XVI)
}

Il suffirait donc de changer les «critères scientifiques » et de conception de ce que l'on appelle une «preuve» pour faire passer l'astrologie de l'état de connaissance opprimée à l'état de véritable science:

\footnotetext{
«chaque fois, on voulut faire rentrer l'astrologie dans le moule des critères classiques de scientificité, et celui de Procuste était chaque fois trop petit, on s'en doute.» (p. 743)

«Tout le problème [...] réside dans l'acception qu'on peut donner du mot preuve, car ce que les astrologues allégueront sous ce nom sera dénié par les scientifiques hostiles à l'astrologie.» (p. XIV)
}

Par ailleurs, si E. Teissier insiste à de nombreuses reprises sur l'absence d'enseignement de l'astrologie à l'université et sur l'absence de département de recherche astrologique au CNRS, c'est bien pour défendre la thèse de la valeur relative de la science actuelle et de l'enseignement tel qu'il est pratiqué. À partir d'un tel argument, fondé sur l'idée de vérité comme pur effet d'un rapport de force, on pourrait tout aussi bien dire qu'en enseignant officiellement l'«art de lire dans les lignes de la main» et en rebaptisant la chiromancie «science de la pré- 
diction des destins individuels » on pourrait imposer un nouvel état de la pensée scientifique, ni plus ni moins valable que le précédent ou que le suivant:

\footnotetext{
«Un fait sociologique surtout nous interpellait: le vide pédagogique de l'astrologie dans les institutions officielles en notre époque. L'intelligentsia semble ignorer en général que cette discipline fut en réalité enseignée à la Sorbonne jusqu'en 1666 et en Allemagne jusqu'en 1821.» (p. XII)

«[Nous nous demanderons] quelles pourraient être les chances de réhabilitation officielle de l'astrologie liée à une situation épistémologique évolutive» (p. 69)

«L'absence de recherches officielles - pour lesquelles il faudrait des subventions de l'Etat -, le refus de prendre en considération le paradigme astrologique, ne serait-ce que pour le réfuter, par exemple au moyen d'un département d'études au CNRS, sont là des symptômes évidents de l'attitude volontairement partiale, omniprésente dans la science officielle.» (p. 762)
}

E. Teissier émet donc des commentaires astrologiques, se livre à une défense de l'astrologie qui est, pour elle, la «reine des sciences » et adopte sans discontinuité le point de vue normatif de l'astrologue plutôt que le point de vue cognitif du sociologue étudiant l'astrologie. Est-ce que, malgré tout, ce point de vue d'astrologue et ce plaidoyer pour l'astrologie s'accompagnent d'une réflexion et d'un travail de recherche sociologiques? L'objet de notre deuxième partie est de montrer qu'il n'en est rien.

\section{LE MAUVAIS TRAITEMENT DE LA SOCIOLOGIE}

Il n'y a, dans le texte d'E. Teissier, aucune trace de problématique sociologique un tant soit peu élaborée, de données empiriques (scientifiquement construites) ou de méthodes de recherche dignes de ce nom. L' «hypothèse» floue annoncée («à savoir cette ambivalence sociétale où prime cependant la fascination, ambivalence qui frise parfois le paradoxe et qui fait figure de schyzophrénie (sic) collective», p. 7) n'est d'ailleurs qu'une affirmation parmi d'autres qui ne débouche sur aucun dispositif de recherche en vue d'essayer de la valider (mais telle qu'elle est formulée, on a en effet du mal à savoir ce qui pourrait être validé ou invalidé).

En revanche, on a affaire, comme nous allons le voir, à de nombreux usages douteux des références sociologiques, à des propos clairement a-sociologiques et anti-rationalistes exprimés dans un style d'écriture pompeux et creux, ainsi qu'à des «données » anecdotiques et narcissiques (E. Teissier à la télévision, E. Teissier et la presse écrite, E. Teissier et ses démêlés avec les scientifiques, E. Teissier et les hommes de pouvoir, Le courrier des lecteurs d'E. Teissier...) suivis de commentaires le plus souvent polémiques (règlements de compte ou récits des règlements de compte avec telle ou telle personnalité de la télévision, tel ou tel scientifique, etc.) ou d'une série de citations d'auteurs rarement en rapport avec les propos qui les précèdent et avec ceux qui les suivent.

\section{Contresens et mauvais usages}

La thèse est truffée de références sociologiques souvent affligeantes pour leurs auteurs (Durkheim, Weber, Berger et Luckmann...) et se lance parfois dans des 
critiques qui montrent que les auteurs critiqués n'ont pas été compris. Il faudrait évidemment des dizaines de pages pour relever chaque erreur de lecture, chaque absurdité, chaque transformation des mots et des idées des auteurs cités et expliquer pourquoi ce qui est dit ne veut rien dire étant donné ce que les auteurs commentés voulait asserter.

Par exemple, le sociologue allemand Max Weber est particulièrement mal traité, systématiquement détourné dans le sens où l'auteur de la thèse a choisi de le faire témoigner. Weber, présenté comme le défenseur d'un «subjectivisme compréhensif» (p. 37) est ainsi inadéquatement invoqué à propos de l'«interactionnisme»:

[À propos des gens qui sont nés le même jour et qui se rendent compte qu'ils ont des points communs]: «On a ainsi des questions du genre: 'Au fait, que vous est-il arrivé en 1978? N'avez-vous pas comme moi divorcé?' Et l'autre de rétorquer: ‘Tiens donc, c'est intéressant. C'est bien fin 1978 que mon couple a connu la crise la plus forte et il est vrai qu'avec ma femme nous avons songé à nous séparer...' À n'en pas douter, ce genre de similitude crée des liens, dans la mesure où l'on se retrouve peu ou prou dans l'Autre et/ou que l'on s'y projette. À travers le dialogue qui s'instaure, on a affaire à un véritable interactionnisme qui, selon Weber, est 'une activité [...] qui se rapporte au comportement d'autrui, par rapport auquel s'oriente son déroulement'» (p. 405-406)

La «sociologie compréhensive» est invoquée à tort et à travers. L'auteur écrit qu'elle va mettre en œuvre «la méthode de la compréhension» (p. VII) en interprétant vaguement la «sociologie compréhensive» comme une sociologie qui donnerait raison aux acteurs (et, en l'occurrence, aux astrologues). Ne pas rompre avec l'astrologie, lui (se) donner d'emblée raison et voir en quoi tout ce qu'on peut lui reprocher est de mauvaise foi: voilà ce qu'E. Teissier comprend du projet scientifique de la sociologie compréhensive appliquée à l'astrologie. Et l'on pourrait faire les mêmes remarques à propos des références à l' «interactionnisme symbolique» dont l'auteur semble à peu près ne connaître que le nom:

«À travers ce que l'on pourrait appeler une herméneutique de l'expérience, c'est la recherche de ce sens, aussi complexe qu'il se révèle, qui sera l'objet du second volet, où nous pratiquerons une sorte d'interactionnisme symbolique (selon l'École de Chicago). Recherche du sens sous-tendu par cette Lebenswelt de l'astrologie, par le donné social, à l'aube de ces temps nouveaux.» (p. 463)

L'on voit aussi se développer les «talents » d'argumentation critique de l'auteur dans ce commentaire de Durkheim, où l'on saisit que l'idée de traiter les faits sociaux comme des choses est «abusive, et donc difficile à admettre parce qu'inadéquate »:

«Dans Les règles de la méthode sociologique, Durkheim affirme que 'les faits sociaux sont des choses'. Encore qu'à coup sûr il faille compter la mouvance astrologique dans les faits sociaux, cette identification, qui consiste à chosifier ainsi un phénomène qui est de l'ordre de l'esprit et du vivant, nous paraît abusive, et donc difficile à admettre parce qu'inadéquate.» (p. 278)

Et que faire, sinon rire, face au drolatique contre-sens sur la pensée de Michel Foucault concernant l' «intellectuel spécifique». L'auteur de la thèse n'ayant de toute évidence pas lu Michel Foucault invoque la soi-disant critique des «intellos spécifiques» (sic) par un Michel Foucault qui justement défendait (en grande partie contre Sartre) la figure de l'«intellectuel spécifique» contre celle d'un 
«intellectuel universel »: «quoique puissent en dire les ‘intellos spécifiques', hostiles au savoir transdisciplinaire, stigmatisés par Michel Foucault» (p. 860).

\section{Des propos a-sociologiques et parfois anti-rationalistes}

On a déjà fait remarquer que l'auteur de la thèse privilégiait le point de vue astrologique sur l'explication sociologique. Mais souvent les explications apportées sont clairement a-sociologiques et trop floues ou trop générales pour être considérées comme de véritables explications. Qu'elle évoque l'«atavisme» ou les «dispositions humaines ataviques » (p. 62), «la part d'ombre» (p. 8) de chacun d'entre nous, la «reliance astrologique intemporelle inscrite au cœur de l'humanité» (p. 62), le «réflexe de l'homme, archaïque et intemporel, universel et omniprésent, qui le porte depuis la nuit des temps à voir une admirable homothétie entre la structure de l'univers et la sienne propre d'une part, la nature qui l'entoure d'autre part» (p. 200), l' «héritage génétique» et le «ciel de naissance» (p. 243), l'«Urgrund commun à toute l'humanité» (p. 253), «la permanence et la similitude de la nature humaine, à la fois sur le plan diachronique et synchronique » (p. 483), E. Teissier explique la fascination des uns et le rejet des autres par la nature humaine, les planètes ou une vague «intuition miraculeuse». Ainsi, commentant les résultats d'un sondage effectué par le journal Le Monde, outre sa polémique avec le journal, E. Teissier se demande face à l'information selon laquelle les femmes seraient plus intéressées que les hommes par l'astrologie: «Faut-il y voir la conséquence d'un syncrétisme ontologique qui la porte à davantage de perméabilité spontanée à tout ce qui est de l'ordre de la Nature, sans la mettre en porte-à-faux avec une intuition qu'elle ne renie pas...» (p. 280). Les exemples de la sorte sont très nombreux:

«faut-il y voir [dans la fascination pour l'astrologie] un souvenir béni de la mémoire col-
lective, une intuition miraculeuse du fil ténu qui le rattache au cosmos et à mère Nature,
comme sa sauvegarde, en somme, en ce monde où une science mécaniste et une société
déshumanisée voudraient le maintenir cloué au sol? Plus concrètement, ce clivage socié-
tal est-il à rapprocher de catégories socioprofessionnelles particulières, de certaines
classes d'âge, de la différence de sexe, ou faut-il le chercher dans l'individu lui-même,
dans sa part d'ombre, quelle que soit sa place dans la société?» (p. 8)

«une recherche des dispositions humaines ataviques et récurrentes pour cette reliance astrologique intemporelle inscrite, semble-t-il, au cœur de l'humanité.» (p. 62)

«on pourrait parler de données immédiates de la conscience collective pour qualifier ce réflexe de l'homme, archaïque et intemporel, universel et omniprésent, qui le porte depuis la nuit des temps à voir une admirable homothétie entre la structure de l'univers et la sienne propre d'une part, la nature qui l'entoure d'autre part» (p. 200)

«En somme, on pourrait comparer l'influence astrale à un vent (cosmique) qui soufflerait dans une certaine direction, induisant des climats et des événements probables, sans préjuger de la réaction de l'homme. Celle-ci étant fonction de sa personnalité propre, elle-même héritée à la fois génétiquement et par le ciel qui l'a vu naître» (p. 243)

«On est ici au cœur d'un anthropomorphisme intemporel, immémorial, atavique, qui participe de cet Urgrund commun à tout l'humanité.» (p. 253)

Mais c'est plus généralement toute explication un tant soit peu rationnelle qui est explicitement rejetée par l'auteur. Devant la trop grande complexité des 
choses, il faudrait abandonner tout espoir de parvenir à en rendre véritablement raison et laisser parler l'intuition sensible et le langage des symboles. Il est vrai que l'auteur est bien aidée dans cette voie par les auteurs qu'elle ne cesse de citer et qui s'affirment assez nettement anti-rationalistes:

« une question primordiale apparaît être la suivante: faut-il voir dans l'approche astrologique une émanation de l'Absolu qui, bien qu'éloignée des religions révélées, serait une tentative humaine pour appréhender, à travers l'ordre cosmique conçu par un Dieu créateur, la manifestation d'une transcendance? Ou bien doit-elle être considérée comme le code explicatif et immanent d'une influence astrale purement physique, phénomène à rapprocher des sciences de la nature? Et dans ce cas, quelle serait la source ontogénétique de cette miraculeuse adéquation universelle, le primum mobile? La réponse à cette question ontologique ne peut qu'être individuelle, car elle se place hors du domaine de la Raison pure, dans celui de l'indémontrable.» (p. 263)

[Citation de Michel Maffesoli] «il convient de dépasser, sans nostalgie aucune, toutes les idéologies se réclamant des prémisses rationalistes (Éloge de la raison sensible, p. 44)» (p. 643)

[Citation en exergue de Michel Maffesoli] «Le rationalisme classique (en sociologie) a fait son temps...» (p. 813)

\section{Refus de toute objectivation}

On aura compris que tout ce qui pourrait permettre d'objectiver et de saisir même partiellement la réalité censée être étudiée est rejeté par l'auteur fascinée, séduite («Simmel étant par ailleurs - et avant tout - un philosophe de la vie, au même titre que Schopenhauer, Bergson ou Nietzsche, cela également était fait pour nous séduire [...]», p. 50) par «la vie» dans toute sa complexité; complexité que les rationalistes, les sociologues positivistes, etc., s'acharnent à vouloir réduire et abîmer. La «méthode» qui convient à un objet aussi complexe et subtil est celle qui est «sensible à l'univers mystérieux, voire insondable, de l'âme humaine». Cette «méthode» est indistinctement désignée par les termes de «méthode phénoménologique», d' «empathie » ou de «sociologie compréhensive»:

«Du fait que l'objectivité parfaite rêvée par la sociologie positiviste se cantonne dans un idéal par définition inaccessible, du fait surtout de la nature de notre sujet, essentiellement complexe, parfois contradictoire dans ses manifestations, un sujet ayant de surcroît pour objet et pour centre un univers subtil où convergent croyance, philosophie, tradition, sacré, affect fait de peur et de fascination, une méthode quantifiante et axée sur des analyses purement rationnelles passerait à côté de la vraie nature du problème. Une autre méthode s'avère donc nécessaire, sensible au monde vécu quotidien d'une part, à l'univers mystérieux, voire insondable, de l'âme humaine d'autre part. S'impose dès lors une méthode phénoménologique qui privilégie l'empathie et ayant pour atout le mérite d'une proximité de l'objet: la méthode dictée par la sociologie wébérienne, bâtie sur la compréhension, prend alors toute sa valeur.» (p. 34-35)

La pensée de l'auteur fonctionne à la façon de la pensée mythique, sans crainte de la contradiction. Pour elle, le «quantitatif» s'oppose au «qualitatif» comme le «carré» s'oppose au «courbe», le «simple» au «complexe» (ou au «subtil»), l'«artificiel» au «naturel», etc. Si elle n'aime pas les méthodes quantitatives, c'est à cause de leur «caractère plaqué et artificiel» (p. 57); si elle n'apprécie pas les statistiques, c'est parce qu'elle sont trop «carrées et linéaires» (p. 295), etc. 
«Utilisant des matériaux aussi variés que parfois difficiles à appréhender parce qu'appartenant davantage à l'univers subtil du qualitatif qu'à celui, mesurable mais tellement moins riche, du quantitatif...» (p. 10)

«Il est néanmoins possible que certains esprits plutôt attachés aux chiffres, aux statistiques et aux faits bruts et simples (encore que l'existence de tels faits soit douteuse et carrément niée par Schutz) puissent trouver cette méthode trop libre, trop fluctuante (ondoyante, dirait Simmel... ou Montaigne!) et subjective, donc manquant de rigueur.» (p. 56)

Mais si les statistiques sont trop grossières pour l'esprit subtil d'E. Teissier, elles peuvent aussi à l'occasion être utiles si on peut leur faire dire des choses positives sur l'astrologie. Par exemple, commentant un sondage sur l'astrologie publié dans Science et vie junior (p. 287-290), elle réagit au fait que les jeunes soient apparemment les plus intéressés par l'astrologie de la manière suivante: «on peut d'ailleurs se demander si cela ne traduit pas un lien avec le cosmos resté plus vivant - et pourquoi pas diraient les adeptes de la réincarnation, un résidu des vies antérieures?» (p. 288). D'un seul coup d'un seul, les pauvres statistiques se transforment, tel le crapaud devenant prince charmant, en preuves irréfutables du sérieux et de la véridicité des analyses astrologiques:

\footnotetext{
«Car nonobstant nos réserves déjà annoncées plus haut, quant à la valeur des analyses quantitatives en général, il reste que des indications chiffrées sur les secteurs de populations adhérant à cette discipline - ou simplement intéressées par cette dernière à divers degrés - ne peuvent que s'avérer des plus précieuses.» (p. 81)

«il y a les statistiques qui sont favorables à l'astrologie d'une façon à la fois péremptoire et éclatante» (p. XV)
}

Et l'auteur se lance parfois elle-même hardiment dans l'évaluation chiffrée, mais totalement intuitive, des faits sociaux : «je pense que ceux qui aujourd'hui en France, font profession d'astrologue et chez qui la spécialité 'astrologie' proprement dite constitue effectivement $90 \%$ et plus de la pratique professionnelle, doivent être moins d'un millier. C'est plus une impression qu'un décompte minutieux, mais ce chiffre me paraît plausible.» (p. 302).

\section{Un étrange discours de la méthode}

Le discours de la méthode chez E. Teissier est aussi précis que ses hypothèses et sa «problématique». Tout d'abord, l'«objectivité» est selon elle un idéal parfaitement inatteignable (un paragraphe entier est consacré au thème de «L'utopie de l'objectivité », p. 28-31). Mais, comme à son habitude, peu hantée par le principe de non-contradiction, E. Teissier peut critiquer la prétention «positiviste» à l'«objectivité» et dire que les scientifiques manquent d'objectivité, ou encore affirmer qu'elle est elle-même animée par un «souci d'objectivité». La question de la possibilité ou l'impossibilité d'une objectivité est donc beaucoup plus complexe que ce qu'un lecteur rationaliste peut modestement imaginer: son sort dépend de la phrase dans laquelle le mot «objectivité » s'insère. Et l'on comprendra que l'auteur revendique l' «objectivité» lorsqu'il s'agit pour elle de défendre l'astrologie:

«Du fait que l'objectivité parfaite rêvée par la sociologie positiviste se cantonne dans un idéal par définition inaccessible [...]» (p. 34) 


\begin{abstract}
«Si l'on veut faire œuvre de sociologue visant à une certaine objectivité, on ne peut donc reprocher à l'art royal des astres, d'être lui aussi, à l'instar de toute croyance, de toute idéologie ou de toute religion, peu ou prou tigrée - pour reprendre le joli titre durandien - de superstition, dans la mesure où cette dernière est omniprésente (et tellement relative au point de vue et à la culture de référence) dans la psyché humaine.» (p. 471)
\end{abstract} «Les points que nous avons cru devoir mettre en relief sont relatifs à la fois à notre
expérience et à notre sensibilité, donc à ce qui nous est proposé et que nous essayons de
communiquer avec un souci d'objectivité. Mais qui peut prétendre à l'objectivité?»
(p. 534)

«Il est vrai que ce déchiffrage ne fut pas toujours aisé, dans la mesure où, étant impliquée nous-même, il nous fallait cependant faire preuve d'un maximum d'objectivité.» (p. 825)

Pour E. Teissier tout est «méthode». Par exemple, lorsqu'elle écrit: «D'où l'importance essentielle de la démarche méthodologique choisie, qui consistera à cerner les motivations et sources secrètes des attitudes et comportements sociaux.» (p. 20), on constate qu'une vague volonté de «cerner des motivations» équivaut pour elle à une «démarche méthodologique». Lorsqu'elle écrit aussi que, dans sa thèse, «la méthode empirique paraît s'imposer» et qu' «elle sera (son) outil de référence» (p. 10), on voit que le mot «méthode», équivalent d' «outil de référence », est utilisé avec l'imprécision la plus grande: "la méthode empirique » semble s'opposer à d'autres «méthodes » (qui ne le sont pas), mais on ne sait pas de quelle méthode précisément il s'agit.

Les termes «méthodes», «paramètres », «facteurs», «outils», etc., sont, en fait, utilisés de manière sémantiquement aléatoire, tant la fonction essentielle de ces usages lexicaux réside dans l'effet savant que l'auteur entend produire sur elle-même et sur le lecteur. Le fait que dans la première citation, E. Teissier dise que les «paramètres» dont elle parle (équivalent ici de «notions») apparaîtront «ici où là, au hasard de cette étude », fait bien apparaître le caractère extrêmement rigoureux de la «démarche méthodologique» mise en œuvre...

\footnotetext{
«Et si les dieux me sont favorables, peut-être pourrons-nous apporter quelques modestes lumières sur l'univers astrologique d'aujourd'hui par rapport à cinq paramètres élémentaires qui, selon NISBET, caractérisent plus que tout autre la sociologie: communauté, autorité, statut, sacré, aliénation, toutes notions qui, ici où là, au hasard de cette étude, la marqueront d'une empreinte en filigrane» (p. 44)

Un grand paragraphe s'intitule «Les outils ou paramètres d'étude» (p. 60-86); «Si l'on vise l'efficacité d'une méthode, celle-ci doit impérativement s'instrumentaliser à travers des outils, ces auxiliaires de la connaissance. Ils nous permettront de prendre en compte les différentes couches de la population concernées par le phénomène astrologique» (p. 60); «Un dernier facteur sera effleuré (car trop important pour être traité en profondeur) avec les rapports de l'astrologie et du pouvoir» (p. 66); «Dans la liste des outils/miroirs de cette investigation...» (p. 83)
}

En sachant tout cela, tout lecteur peut mesurer l'effet comique de la prétention toute verbaliste à la rigueur qu'affiche l'auteur de la thèse: "nous avons eu l'occasion de développer l'esprit de rigueur dont l'exigence nous habite depuis toujours. À cela s'ajoutait un souci de rationalité, de cohérence, mais cela à travers une forte curiosité intellectuelle au service d'une recherche de la vérité » (p. VIII). Visiblement, l'esprit ne parvient pas à guider les gestes. 


\section{Les «données»: anecdotes de la vie personnelle, médiatique et mondaine d'Élizabeth Teissier}

Si l'on entend par «données empiriques » des matériaux qui sont sélectionnés, recueillis et/ou produits en vue de l'interprétation la plus fondée possible de tel ou tel aspect du monde social, c'est-à-dire à des corpus de données dont les principes de constitution et de délimitation sont explicitement énoncés, on peut dire sans risque que la thèse d'E. Teissier ne contient strictement aucune donnée empirique. Si l'auteur avait une conception un tant soit peu empirique de la pratique de recherche en sociologie (rappelons qu'elle dit mettre en œuvre «la méthode empirique »), elle n'oserait par exemple pas écrire avec autant de légèreté et d'inconscience empirique qu'elle va suivre l'évolution de l'astrologie «à travers le temps et l'espace dans les sociétés les plus diverses, de la nuit des temps à nos jours » en annonçant explicitement qu'elle se livrera «à un rapide survol, aussi bien chronologique que géographique, diachronique que synchronique...» (p. 93). Mais pourquoi se donner la peine de mettre en place un véritable dispositif de recherche lorsque l'on pense que «la vitalité de l'astrologie aujourd'hui ne fait aucun doute» et que «pour preuve, il suffit d'ouvrir les yeux et les oreilles» (p. 792)?

De même, comment apporter une preuve de «l'intérêt de plus en plus marqué des médias pour l'astrologie »? E. Teissier répond: «il n'y a pas une semaine où nous ne soyons pas sollicitée à participer, ici ou là, en France ou à l'étranger, à une émission de ce genre» (p. 274). En fait, E. Teissier enchaîne de manière aléatoire les anecdotes personnelles au gré de l'association de ses souvenirs: "Dans le contexte de l'être-ensemble, une autre histoire nous revient à l'esprit, où nous étions à la fois témoin et partie» (p. 412); «Une autre histoire exemplaire nous revient à l'esprit.» (p. 383), etc. Elle raconte ce qu'on lui a dit ou écrit et ce qu'elle a répondu. Ses commentaires, quand il y en a, se contentent de prolonger la polémique lorsqu'il y avait polémique (avec les journalistes, les animateurs de télévision, les scientifiques, etc.) et de souligner l'intérêt pour l'astrologie - malgré le consensus culturel en défaveur de l'astrologie et la ghéttoïsation de cette dernière - qu'illustrent certaines anecdotes. L'anecdote tirée «au hasard» (signe sans doute d'objectivité à ses yeux) fait toujours preuve:

\footnotetext{
«Nous pourrions allonger indéfiniment la liste de ces lettres qui sont autant de témoignages du puissant impact sociétal de l'astrologie aujourd'hui » (p. 345)

«Pour montrer que l'impact de l'astrologie n'a pas de frontières et s'exerce en priorité sur les jeunes intellectuels, mentionnons encore cette lettre qui nous parvint...» (p. 354)

«nous avons tenté de dresser un panel évocateur des diverses activités sociétales reflétant l'attraction des diverses couches de la société contemporaine pour l'astrologie...» (p. 462)

«Parmi les éléments qui tendraient à refléter la notion de fascination, on peut estimer que le simple fait que le président des Français ait fait appel à nous est en soi signifiant.» (p. 440)

«À travers ces quelques expériences exemplaires, choisies au hasard dans notre parcours,
nous retrouverons des Leitmotive qui se répondent en contrepoint, manifestations quasi
permanentes, voire endémiques du rejet socioculturel, du tabou de l'astrologie aujour-
d'hui.» (p. 544)
}

Si elle fait également le compte rendu d'échanges de courriers avec certains lecteurs, pour «preuve» de l'ambivalence fascination/rejet vis-à-vis de l'astrolo- 
gie ( «C- Le courrier des lecteurs et téléspectateurs, baromètres de notre société », p. 311-386), il n'est aucunement question de constituer un corpus, ni même de faire une analyse sociologique, mais de donner à lire le courrier reçu, ainsi que les réponses envoyées («Voici ce que nous avons répondu à ce lecteur:...», p. 319; «Voici la réponse que nous adressâmes à cette lectrice désorientée », p. 327). On n'a pas même d'évaluation précise des différents types de courriers qu'elle reçoit. Ainsi, à propos des lettres qu'elle range dans la rubrique «Les appels à l'aide», elle écrit de manière approximative: "Il s'agit certainement, quantitativement parlant, de la masse la plus importante de lettres reçues» (p. 312) ou encore que «Parmi les appels à l'aide, les lettres émanant de prisonniers ne sont pas rares » (p. 321).

Et l'on va ainsi d'une anecdote à l'autre: E. Teissier en «face-à-face avec un astronome monolithique dans son agressivité»(p. 543), E. Teissier et Marcel Jullian, PDG d'Antenne 2 (p. 588-629) à propos de l'émission Astralement vôtre, E. Teissier et l'émission allemande Astrow-show entre 1981 et 1983 (p. 645 et suivantes), E. Teissier et l'émission Comme un lundi de Christophe Dechavanne du 8 janvier 1996 (p. 671-685), E. Teissier et l'émission Duel sur la cinq du 10 juin 1988 (p. 709-725), etc. Et à chaque fois, l'auteur émet des jugements péremptoires, polémique, formule des réponses agressives. Elle n'étudie donc pas les réactions à l'astrologie, elle la défend. Elle ne fait pas l'analyse des polémiques autour de l'astrologie, mais est dans la polémique, continuant dans cette thèse comme sur les plateaux de télévision, sur les ondes radiophoniques ou dans la presse écrite - à batailler contre ceux qui considèrent que ce n'est pas une science.

Dans tous les cas, le narcissisme naïf est grand, bien que totalement dénié: «Bien que nous refusions dans ce travail de nous mettre en avant pour des raisons à la fois d'objectivité et d'une décence de bon aloi, on aura remarqué que nous fûmes à travers toute l'émission la seule astrologue à être prise à parti...» (p. 686). Non seulement les exemples pris par E. Teissier ne concernent qu'E. Teissier (alors même qu'elle aurait pu s'intéresser à d'autres collègues astrologues), mais les récits mettent toujours en avant la vie héroïque ou passionnante d'E. Teissier. C'est ainsi qu'elle raconte par exemple comment la rencontre de l'astrologie fut «le grand tournant de sa vie»: «Nous eûmes droit à notre nuit de Pascal - nuit boréale en réalité, car l' illumination' dura quelque six mois, le temps d'apprendre les fondements cosmographiques et symboliques de l'art royal des astres $^{16}$, suffisamment pour être éblouie des 'convergences' d'une part psychologiques, d'autre part événementielles avec notre caractère et notre vécu, ou ceux de notre entourage» (p. X). Ou encore, faisant le récit du contexte dans lequel elle a été contactée pour présenter l'émission allemande Astro-Show: «Lorsque, au tout début de 1981, à notre retour d'un voyage en Inde, nous trouvâmes trois messages consécutifs et quelque peu impatients de l'ARD (première chaîne télévisuelle allemande), nous fûmes plutôt surprise. Jusque-là en effet notre rayon d'action n'avait pas passé les limites du Rhin.» (p. 646).

Le narcissisme rejoint l'indécence lorsque, après avoir donné à lire le courrier d'un lecteur, jeune maghrébin incarcéré aux Baumettes, elle écrit avec misérabi-

16 Le lecteur aura noté au passage qu'une formation d'une durée de six mois permet d'acquérir «les fondements cosmographiques et symboliques de l'art royal des astres ». L'effort n'est finalement pas si considérable que cela pour pouvoir «écouter la musique» des planètes. 
lisme et condescendance et sans prendre conscience du ridicule: «Nous étions consciente à quel point le fait de dialoguer pouvait être important pour ce jeune homme visiblement égaré, tel un zombie, en ce bas monde. [...] Voici la teneur de notre réponse audit prisonnier: 'Cher Eric, je ne trouve hélas le temps de répondre à votre lettre qu'au retour d'un séjour à l'étranger. Je vous envoie une analyse à titre tout à fait exceptionnel, et pour la somme purement symbolique d'un franc (vu votre situation actuelle). Votre thème me montre que votre futur florissant vous permettra de m'envoyer ce que bon vous semblera en temps venu. Sachez que vous devez ce cadeau d'une part à vos bonnes étoiles [...], d'autre part au fait que votre lettre m'a touchée, également du fait que j'ai deux filles, l'une Poisson, l'autre née en 1973, comme vous !... Je vous demande en revanche de bien vouloir en garder le secret, puisque l'ébruiter pourrait faire des jaloux [...]'» (p. 323).

\section{Une écriture boursouflée et creuse}

Le problème essentiel avec le style d'écriture que l'on trouve dans une thèse comme celle d'E. Teissier, réside dans le fait que l'on aura beau multiplier les «échantillons», répéter les citations en vue de prouver que l'on a affaire à une écriture jargonnante, peu rigoureuse, souvent incompréhensible, parfois proche de l'absurde, d'autres verront au contraire dans les mêmes extraits toutes les marques de la profondeur ou de l'intelligence du propos. Question d'habitude pourrait-on dire. S'il est habitué à un usage déréglé de la langue, le lecteur appréciera les acrobaties verbales ou les associations aléatoires d'idées un peu comme s'il était devant un grand poème pseudo-savant. Mais là réside exactement le problème: le sociologue n'est pas un poète. On peut apprécier les poèmes de René Char ou de Stéphane Mallarmé et ne pas accepter, en matière de sciences sociales, ceux qui s'acharnent à faire dans le creux et le vague en faisant passer le creux pour du profond et le vague pour du riche et du complexe. Devant un grand nombre de passages de cette thèse, nous pourrions émettre le jugement suivant: dans la mesure où nous croyons savoir ce que parler en sociologue veut dire, nous pouvons témoigner du fait que nous n'avons rien compris à ce qui a été dit. Mais qu'y aurait-il à comprendre lorsque rien n'a été vraiment dit?

Que des sociologues en France aient contracté de mauvaises habitudes de parler et d'écrire, qu'ils tentent de les transmettre régulièrement à des centaines, voire à des milliers d'étudiants, cela constitue un fait social majeur. Cependant, on peut avoir acquis auprès de maîtres, prestigieux ou moins prestigieux, de telles mauvaises habitudes ${ }^{17}$, et vouloir néanmoins s'en débarrasser une fois qu'on en a pris conscience. Mais encore une fois ce que l'on peut appeler «mauvaise habitude de parler et d'écrire », à partir d'une conception un tant soit peu rationaliste de l'argumentation, est malheureusement perçue par beaucoup de ceux qui les mettent en œuvre comme un signe d'intelligence et de pensée originale et profonde. L'écriture et la parole déréglées peuvent ainsi se transmettre de génération en génération, sans que ceux qui transmettent ni ceux qui se les approprient ne sachent exactement de quoi ils parlent, dans l'obscurité de la relation enchantée de maîtres à élèves (ou disciples) où se constituent ordinairement de telles habitudes.

17 Sur les habitudes discursives et mentales voir B. Lahire, L'Invention de l' «illettrisme». Rhétorique publique, éthique et stigmates, Paris, Éditions la Découverte, Coll. «Textes à l'appui», 1999. 
Il suffit d'être fasciné, de trouver cela beau, profond, sensible, original, pour avoir envie de faire de même et paraître à son tour intelligent, original, sensible et profond. Le sens de tout cela peut très bien être totalement absent des mots qui s'écrivent et s'échangent et se situer exclusivement dans la relation fascinée au maître. Peu de signification discursive, beaucoup de signification sociale.

Délire sémantique ou esbroufe verbale, plaisir des mots savants qui sonnent bien accolés les uns aux autres pour asserter des banalités sur un ton sérieux, enchaînements des citations d'auteurs aussi ésotériques les unes que les autres, la panoplie de l'écriture pseudo-savante et réellement floue est assez complète. Donnons-en quelques exemples en garantissant au lecteur que l'effet d'étrangeté n'est pas le produit d'une injuste décontextualisation:

«[...] à la recherche de cet infracassable noyau de nuit qui hante le cœur de l'homme depuis des temps immémoriaux?» (p. 7)

[M. Maffesoli parle du «sentiment cosmique» qui «incline vers le vrai organique, le vivant, c'est-à-dire vers le naturalisme»] «Ce sentiment cosmique se trouvant de plus en plus impliqué comme trame de fond dans notre recherche, dont il constitue en quelque sorte le tissu, il allait de soi qu'il serve aussi de reliance et de sésame heuristique dans notre démarche, c'est-à-dire, dit concrètement, de fil rouge instrumental au service de notre projet de décrypter le donné social de ce que Dilthey appellerait la structure psychique que constitue l'astrologie.» (p. 49)

«Ainsi, comme lanterne magique pour nous guider à travers ces méandres heuristiques, nous utiliserons, comme accessoire à la raison objective et raisonnante la raison sensible maffésolienne ou l'intuition intellectuelle d'un R. Guenon, telle qu'elle est évoquée par F. Bonardel.» (p. 53)

«Au-delà de cette complexité qui ne doit pas être un mot refuge, nous cultivons l'espoir - l'utopie? - qu'en fin d'analyse, ayant, même imparfaitement, décrypté les arcanes de notre problématique, nous pourrons adhérer pleinement à l'affirmation du sociologue lorsqu'il déclare que la complexité est 'le défi à affronter, ce qui aide à le relever, et parfois même à le surmonter' (E. Morin, Introduction à la pensée complexe)» (p. 68)

«En tout état de cause, on assiste ici à un triple trajet - ou trajectorialité, au sens durandien -, dans un va-et-vient simmélien qui s'inscrit en premier lieu entre le consultant et le système astrologique» (p. 76)

«Mais on trouve également un autre vecteur de cette trajectivité, à savoir celui entre ce même acteur social et la réelle présence (pour reprendre un expression chère à G. Steiner) du consulté, de l'astrologue en l'occurrence. En effet, il s'agit bien de la manifestation symbolique d'un présentéisme dépouillé de tout jugement moral, où seul intervient le laisser-être, à l'exclusion d'un aspect quelconque de contrainte, d'un devoir-être». (p. 77)

«Ne doutons pas que c'est dans cette effervescence que se trouve la vérité sociétale. Et cette effervescence se focalise de plus en plus sur le monde des astres, nous l'avons, pensons-nous largement montré au cours de notre survol. On assiste en effet de plus en plus et dans tous les domaines du quotidien à une infiltration diffuse et effervescente de l'astral en nos sociétés postmodernes, en résonance, quelque part, à la coupure épistémologique, déjà évoquée, d'une postmodernité dont Maffesoli donne la définition suivante: 'Sorte d'agglutination, à la fois disparate et tout à fait unie, d'éléments les plus divers', impliquant un 'style organique', ce dernier se révélant 'une bonne manière d'appréhender la raison interne d'une connaissance' (Préface à Durkheim, Les Formes élémentaires de la vie religieuse, p. 90)» (p. 851)

«Tout au long de notre thèse, nous avons à l'instar de ce qui est la vocation et l'objectif du chercheur, tenté de déceler les prémices sous-jacents, les frémissements de ce qui est 


\begin{abstract}
'en train de naître' et qui se font sentir dans la réalité sociétale aujourd'hui. Cela en pratiquant ce que G. Durand appelle une 'pensée concentrique', c'est-à-dire une 'pensée formant un système ouvert qui refuse de rester au centre mais qui va glaner ce qui se passe et se propage en périphérie à la recherche de l'humus sous-jacent'. Autrement dit, il s'agissait de suivre un processus de va-et-vient, en vases communiquants, tout en refusant de rester prisonnier d'une idée, d'aller à la rencontre de l'inconnu, de ce qui se vit dans le donné social, de ce qui émerge dans le champ expérimental du chercheur. De tout ce vécu, de cet observé, nous avons tenté de dégager la dynamique à travers une synergie de la pensée, en délaissant son contraire: la pensée unique, sous forme d'une doxa synonyme d'apparence. Nous avons ainsi pu faire état de ce maillage multiple, de ces innombrables passerelles qui s'effectuent entre échanges de savoirs, dans un désir commun de s'ouvrir à d'autres connaissances et de partager son intérêt, mais aussi à travers ces nouvelles technologies, longuement évoquées, où tout un chacun fait un pied-de-nez à cette pensée conformiste représentée par ceux qui détiennent un pseudo-savoir - un 'demisavoir' selon J.-C. (sic) Domenach. Au fil de notre travail, nous avons pu mettre le doigt sur la confusion qui émerge par rapport à ces données, où sont mis à mal ceux qui croyaient détenir le savoir, cette pensée bien gardée, convenable, intellectuellement correcte, tout en montrant que son impérialisme peu à peu se désagrège - et ce en dépit d'un combat d'arrière-garde qui se voit voué à un échec à long terme. Comme nous avons montré, pensons-nous, l'inanité d'un intellectualisme desséché. 'Le règne absolu de l'idée ne peut s'établir ni surtout se maintenir: car c'est la mort' (in Le suicide de Durkheim cité par Maffesoli dans sa préface aux Formes élémentaires de la vie religieuse, p. 11). En paraphrasant K. Jaspers, on pourrait dire que 'c'est dans la communication qu'on atteint le but de l'astrologie (la philosophie)' (Introduction à la philosophie, p. 25), dans cet échange chaleureux (dionysiaque?) entre esprits branchés sur des intérêts semblables, orientés en l'occurrence sur les arcanes célestes.» (p. 861)
\end{abstract}

\title{
CE QUI NUIT RÉELLEMENT À LA DISCIPLINE
}

«À Londres, s'il n’y a pas non plus de chaire d'astrologie à l'université, du moins peut-on obtenir un P.H.D. (doctorat) en astrologie si cette discipline est officiellement dépendante de la branche Psychologie; même biais possibles en France à condition de choisir un sujet de thèse limitrophe de la Sociologie, de la Philosophie ou de l'Histoire des religions et de se placer officiellement sous l'égide ces disciplines: on est obligé de camoufler, de tricher, de contourner les institutions qui sont manifestement en retard sur la réalité d'un consensus de plus en plus évident.» (p. 815-816). La vérité de la thèse d'E. Teissier est très clairement énoncée dans ces pages. Pour défendre la cause de l'astrologie, il faut avancer masqué: philosophie, histoire de religion ou sociologie peuvent être des entrées possibles pour l'astrologue. Mais si de telles stratégies de légitimation peuvent être imaginées, c'est que notre communauté scientifique les rend possibles. Et c'est cela qui pose fondamentalement problème au-delà du cas particulier de cette thèse. Que les choses soient claires: E. Teissier ne peut être tenue pour responsable de ce qui s'est passé à la Sorbonne et elle n'aurait pas même eu l'idée de frapper à la porte de notre discipline pour trouver un lieu de légitimation de ses propres intérêts d'astrologue, si celle-ci n'était pas le refuge d'enseignants-chercheurs dépourvus de rigueur et parfois très explicitement anti-rationalistes.

Revenons à notre point de départ: des «collègues » (abondamment cités dans cette thèse) ont délivré un droit de soutenance à l'auteur de cette thèse, puis, avec d'autres, ont décidé de lui attribuer la mention «Très honorable». Après lecture du 
compte rendu précédent, on comprend à quel point le sentiment de scandale du lecteur de la thèse est grand.

Évidemment, le directeur de la thèse, Michel Maffesoli, est Professeur de sociologie à l'université de Paris $\mathrm{V}$ et a même été promu à la première classe par le $\mathrm{CNU}$, il publie régulièrement des ouvrages, préface des classiques de la sociologie, dirige une revue et fait soutenir des thèses à un rythme particulièrement élevé $^{18}$, etc. Il soutient, avec l'aplomb cynique de celui qui sait pertinemment que la thèse ne sera pas lue intégralement par les étudiants ni même par les sociologues professionnels qui ont généralement d'autres tâches plus urgentes à faire, que la thèse d'E. Teissier est une thèse «sur l'astrologie» (à quelques «dérapages » près avoue-t-il ${ }^{19}$, en rajoutant de manière insultante pour tous les sociologues qui font leur travail d'évaluation des thèses sérieusement: «En toute honnêteté, lequel d'entre nous, directeur de thèse n'a pas laissé passer de tels 'dérapages'?») et non une «thèse d'astrologie». Il peut donc utiliser l'argument du meurtre d'une école de pensée ${ }^{20}$ ( «Il ne faudrait pas que cette thèse serve de prétexte à un nouveau règlement de compte contre une des diverses manières d'envisager la sociologie.» ${ }^{21}$ ) et dénoncer la «chasse à l'homme» qui est lancée contre lui: «Est ce que cette thèse n'est pas un simple prétexte pour marginaliser un courant sociologique, et disons le crûment, pour faire une chasse à l'homme, en la matière contre moi-même?» ${ }^{22}$ Et c'est bien comme cela que certains collègues ont interprété les réactions négatives à cette soutenance de thèse et à l'attribution d'un titre de docteur en sociologie ${ }^{23}$. Or, il n'est bien sûr pas question de querelles d'écoles dans cette affaire, mais de rigueur scientifique (et même, plus largement, de rigueur intellectuelle) et de définition du métier de sociologue.

Les véritables questions au fond que pose une telle «affaire» nous semblent être les suivantes: Comment parvenir à transformer collectivement les produits

18 Lorsque l'on compte le nombre de thèses de doctorat soutenues par directeur de thèse, en France entre 1989 et 1995, on s'aperçoit que Michel Maffesoli arrive très largement en tête des directeurs de thèse avec 49 thèses (en 7 ans, soit en moyenne 7 thèses par an) soutenues, loin devant LouisVincent Thomas (33). Puis viennent Pierre Ansart (22), Jean Duvignaud (22), Pierre Fougeyrollas (19), Raymond Bourdon (17), Annie Kriegel (14), Alain Touraine (14), Jacques Lautmann (12), Robert Castel (11), Jean-Michel Berthelot (10) et Roger Establet (10).

19 M. Maffesoli, courriel daté du 23. 04. 01 adressé à de nombreux sociologues.

20 Et ce d'autant plus qu'on a contribué à banaliser sa présence au sein de la discipline dans des manuels universitaires « respectueux de la pluralité des écoles et des sensibilités », comme le mentionne la quatrième de couverture de l'ouvrage La Sociologie française contemporaine (sous la direction de J.-M. Berthelot, Paris, PUF, 2000); ouvrage qui comprend un chapitre, signé par Patrick Tacussel, intitulé «La sociologie interprétative. Un tournant postempiriste dans les sciences humaines en France», p. 117-125.

21 Lettre de M. Maffesoli adressée par courrier électronique le 23. 04. 01. Une autre variante se retrouve dans le texte accompagnant le courrier: «Une question de bon sens se pose, est-ce que, finalement, cette thèse non lue, n'est pas prétexte à règlement de compte contre un type de sociologie que je représente?»

22 M. Maffesoli, courriel daté du 25. 04. 01 adressé à de nombreux sociologues.

23 On a pu lire ainsi dans la presse qu' «Elizabeth Teissier pourrait devenir le pion qu'on avance en surface pour régler des affaires plus souterraines, relevant des querelles de chapelle ou des jeux de pouvoir entre 'grands' de la sociologie» (O. Piriou, «Banalité d'Elizabeth Teissier», Le Monde daté du 30.04. 01). 
d'une histoire (académique et scientifique) mal faite (attributions abusives du titre de docteur en sociologie, recrutements universitaires peu rigoureux, revues scientifiques à faible contrôle scientifique...)? Comment justifier, sans apparaître injuste et terroriste, l'affirmation selon laquelle Michel Maffesoli (entre autres) n'est pas sociologue et n'est pas en mesure de former les étudiants dont il dirige les travaux de recherche au métier de sociologue? Ce sont ces questions que les sociologues doivent affronter. Sans prise de conscience collective de notre communauté scientifique, il n'y a aucune raison que ce genre de faits ne se renouvelle pas à l'avenir, avec moins de fracas, car tous les candidats n'auront pas l'honneur de la grande presse.

«Mais se servir de cette thèse, pour régler des comptes, pour sonner l'hallali, ne me paraît pas sain et, en tout cas, risque de nuire à notre discipline, en général », écrit Michel Maffesolii ${ }^{24}$. Nous espérons avoir contribué ici à montrer que ce qui «nuit à notre discipline » et ce qui n'est «pas sain», c'est très précisément le genre de spectacle dont la Sorbonne a été le théâtre sous la responsabilité d'un jury en grande partie composé de sociologues. Personnage cynique ${ }^{25}$, fin stratège, maniant habilement l'art du renversement des situations, Michel Maffesoli voudrait nous faire croire que «les fautifs ne sont pas ceux qui commettent les fautes [...], mais ceux qui ont l'impudence de les dénoncer ${ }^{26}$. Gageons que les diverses réactions saines à cette affaire malsaine puissent donner l'occasion d'une réflexion collective sur le métier de sociologue et sur les conditions d'entrée dans ce métier.

Ecole Normale Supérieure

Lettres et Sciences Humaines

Lyon

24 M. Maffesoli, courriel daté du 25. 04. 01, op. cit. Voir aussi sa réponse à l'article de Jean Bricmont et Diana Johnston («Le monde diplomatique», août 2001): «L'astrologie, la gauche et la science», in «Le monde diplomatique », 2 octobre 2001, p. 2.

25 M. Maffesoli a qualifié, de manière tout à fait insultante, l' «ennuyeux jargon» des sociologues d' «argot de proxénètes » dans la préface au livre de Alfred Schutz, Le Chercheur et le quotidien, Paris, Méridiens Klincksieck, 1987, p. I.

26 J. Bouveresse, Le Philosophe chez les autophages, Paris, Minuit, «Critique», 1984, p. 114. 